\title{
Vibration Control of Cantilever Beams Moving Along the Axial Direction
}

Etsujiro IMANISHI

\author{
Mechanical Engineering Research Laboratory \\ KOBE STEEL, LTD.Kobe, Hyogo, 651-2271, \\ JAPAN \\ Email: e-imanishi@rd.kcrl.kobelco.co.jp
}

\author{
Naoki SUGANO \\ Mechanical Engineering Research Laboratory \\ KOBE STEEL, LTD.Kobe, Hyogo, 651-2271, \\ JAPAN \\ Email: n-sugano@rd.kcrl.kobelco.co.jp
}

Keywords: Vibration of Cantilever Moving Beams, Self-exciting Vibration, Finite Element Method,Multibody Dynamics

\begin{abstract}
In this paper a vibration control technique is proposed for flexible body moving along the axial direction such as the plates or the wire rods in the mill, and the effect is verified by numerical simulation. The dynamic characteristics of the cantilever beam moving along the axial direction are examined by using the Lagrange's equation of motion. It is shown that the vibration of the cantilever beam moving along the axial direction is caused by self-excited vibration, and that optimal damper-spring properties at the beam support point are obtained by examining the stability of a simple vibration model.
\end{abstract}

\section{Introduction}

In the rolling process of the plates and the wire rods, the high speed rolling is required for the improvement in productivity in recent years. The vibration, however, occurs in the rolling materials on high-speed rolling, the injuries are given in the products and it causes the dimensional accuracy problems getting worse. Especially the violent vibration of the rolling material occurs when the termination is drawn into the rolling mill. The problem is called 'spaghetti problem', that is observed when the flexible body is drawn into a constraint region, and the vibration control technique has been required. Various researches concerning the vibration analysis technique have been carried out. For example, Stolte and Benson[1] studied the vibration problem for the paper sending on a high-speed copy machine. Sugiyama and Kobayashi[3] carried out the modeling for this problem, that many small rigid bodies and rotation springs were combined with, and made the comparison experimental results with the modeling. On the other hand, authors[4] proposed the dynamic analysis technique using the finite element formulation that 2dimensional beams considering the speed moving along the axial direction were modeled.

In this paper a vibration control method is proposed for the system. Firstly, it is shown that the unstable phenomenon by divergence occurs as the speed becomes large, while the dynamic characteristics of the beam moving along the axial direction are examined by simple modeling. Secondly the stability of the system is examined for the damper-spring property at the beam support point, and the optimal damperspring properties for stabilizing the system are obtained. Furthermore, while the vibration control method is proposed for reducing the vibration using the examination, it is shown that the outstanding vibration reduction effect is obtained by conducting the simulation using the detailed model on the finite element formulation.

2. Dynamic analysis of a cantilever beam moving along the axial direction using finite element formulation

The vibration of the rolling material occurs when the termination is drawn into the rolling mill as shown in Fig.1. This research examines the vibration by modeling as a 2 dimensional beam moving along the axial direction. The authors presented the modeling technique using a finite element formulation in previous report [4]. Therefore, this method is briefly explained here, and the simulation examples of the vibration are shown in this section. 


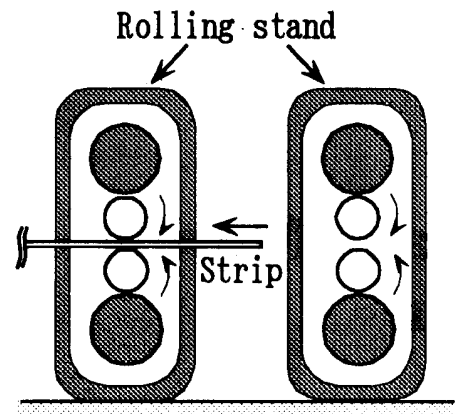

Fig.1 Dynamics in the rolling process

\subsection{Equation of motion for 2-dimensional beam moving along the axial direction}

Fig.2 shows the model of the 2-dimensional beam moving along the axial direction. In this model, the beam is divided into $\mathrm{N}$ elements. The element coordinate system of the $\mathrm{i}$-th element in this model is shown in Fig.3. The local reference frame is taken so as the axis $x^{\prime}$ to be agree with the tangential direction of the element in the node $A$. The displacement vector in the element coordinate system at the arbitrary point, the node $\mathrm{A}$, and the node $\mathrm{B}$ after deformation are expressed as the followings, respectively

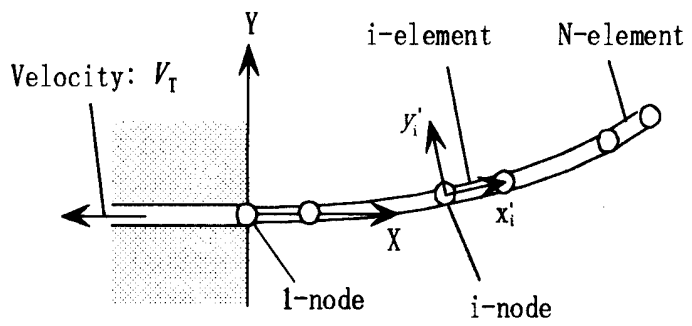

Fig.2 Cantilever beam model moving along the axial direction

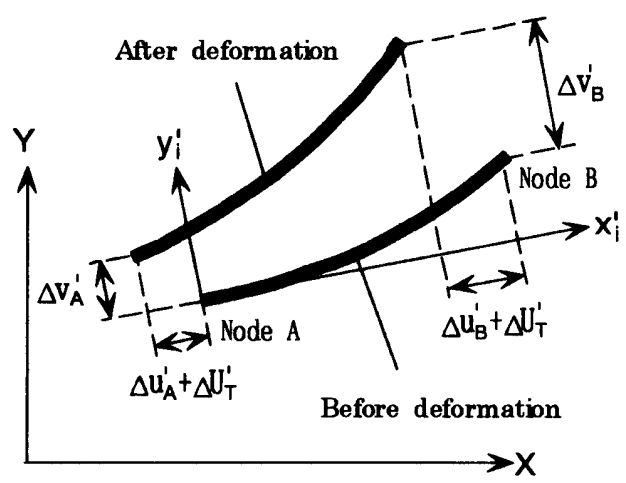

Fig.3 Coordinate system of beam element $i$

$$
\begin{aligned}
& \Delta u_{A}^{\prime}+\Delta U_{T}^{\prime}=\left[\Delta u_{A}^{\prime}, \Delta v_{A}^{\prime}, \Delta \theta_{A}^{\prime}\right]^{T}+\left[\Delta U_{T}^{\prime}, 0,0\right]^{T} \\
& \Delta u_{B}^{\prime}+\Delta U_{T}^{\prime}=\left[\Delta u_{B}^{\prime}, \Delta v_{B}^{\prime}, \Delta \theta_{B}^{\prime}\right]^{T}+\left[\Delta U_{T}^{\prime}, 0,0\right]^{T} \\
& \Delta u_{0}^{\prime}+\Delta U_{T}^{\prime}=\left[\Delta u_{0}^{\prime}, \Delta v_{0}^{\prime}, \Delta \theta_{0}^{\prime}\right]^{T}+\left[\Delta U_{T}^{\prime}, 0,0\right]^{T},
\end{aligned}
$$

where $\Delta U_{T}^{\prime}$ is the displacement vector generated by the motion of the whole beam in the axial direction. The relation between $\Delta u_{0}^{\prime}$ and $\Delta u_{A}^{\prime}, \Delta u_{B}^{\prime}$ is expressed using the shape function $N$ as follows

$$
\Delta u_{0}^{\prime}=N\left(\Delta u_{A}^{\prime}, \Delta u_{B}^{\prime} T\right)^{T}=N \Delta q^{\prime} \text {. }
$$

Transforming $\Delta u_{\mathrm{A}}^{\prime}, \Delta \boldsymbol{U}_{\mathrm{T}}^{\prime}, \Delta u_{0}^{\prime}, \Delta q^{\prime}$ to displacement vectors in a global coordinate system $\Delta u_{\mathrm{A}}, \Delta u_{\mathrm{B}}, \Delta u_{T}, \Delta u_{0}, \Delta q$, and solving velocity vectors at arbitrary points by differentiating with respect to time, the velocity vectors are substituted into Lagrange's equation of motion (3),(4).

$$
\begin{aligned}
& \frac{d}{d t}\left(\frac{\partial L}{\partial \Delta \dot{q}^{T}}\right)-\frac{\partial L}{\partial \Delta q^{T}}=0 \\
& L=\frac{1}{2} \rho \mathrm{A} \int_{0}^{l}\left(\Delta \dot{u}_{0}^{T} \Delta \dot{u}_{0}+\Delta \dot{U}_{T}^{T} \Delta \dot{U}_{T}\right) d x^{\prime}-\Pi_{s}-\Pi_{f} \\
& =\frac{1}{2} \rho \mathrm{A} \int_{0}^{l} \Delta \dot{u}_{0}^{T} \Delta \dot{u}_{0} d x^{\prime}+\frac{1}{2} \rho A l V_{T}^{2}-\Pi_{s}-\Pi_{f}
\end{aligned}
$$

where $\rho$ is the mass density of the beam, $A$ is the section area, $V_{T}$ is speed of the whole beam, $\Pi_{\mathrm{s}}$ is the strain energy as follows

$$
\Pi_{s}=\int_{0}^{l}\left(\frac{E A}{2} \varepsilon^{2}+\frac{E I}{2} \kappa^{2}+\frac{G A}{2} \gamma^{2}\right) d x^{\prime}
$$

( $\varepsilon:$ axial strain, $\kappa:$ curvature, $\gamma:$ shear strain) and $\Pi_{\mathrm{f}}$ is the potential of applied force as follows

$$
\begin{aligned}
& \Pi_{f}=F^{T} \Delta q, \\
& F=\left(F_{x A}, F_{y A}, M_{\theta A}, F_{x B}, F_{y B}, M_{\theta B}\right)^{T},
\end{aligned}
$$

where $F_{x}, F_{y}, M_{\theta}$ are the force in $x$ direction, $y$ direction, and moment respectively.

By arranging the above equation, the incremental equations of motion are finally derived as follows:

$$
\begin{aligned}
{\left[M_{\theta}\right] \Delta \ddot{q}+\left(\left[C_{\mathrm{v}}\right]-\left[C_{\mathrm{v}}\right]^{\mathrm{T}}+\left[\dot{M}_{0}\right]\right) \Delta \dot{q}+} & \left(\left[K_{0}\right]+\left[K_{G}\right]+\left[K_{\mathrm{v}}\right]+\left[\dot{C}_{\mathrm{v}}\right]^{\mathrm{T}}\right) \Delta \boldsymbol{q}=\Delta \boldsymbol{F} .
\end{aligned}
$$

where $\left[M_{0}\right]$ is derived by transforming a mass matrix $\left[M_{B}\right]$ of the linear beam element to global coordinate. $\left[\dot{M}_{0}\right]$ is the time derivatives, that is caused by varying the beam element length with respect to time as mentioned above. $\left[K_{0}\right]$ and $\left[K_{G}\right]$ in Eq.(8) are derived by transforming a stiffness matrix $\left[K_{B}\right]$ of the linear beam element and a geometrical stiffness matrix $\left[K_{G B}\right]$ to global coordinate system, that are derived by using the displacement-strain relation considering geometric nonlinearity in the strain of Eq.(5).

$\left[C_{v}\right],\left[K_{v}\right]$ in Eq.(8) are the terms caused by the motion of the beam along the axial direction as follows

$\left[\boldsymbol{C}_{\mathrm{v}}\right]=\left[\boldsymbol{C}_{1}\right]+\left[\boldsymbol{C}_{2}\right]$

$\left[C_{1}\right]=\rho A i D^{T} \int_{0}^{d} N^{T} N_{l} d x^{\prime} D$ 


$$
\begin{aligned}
& {\left[C_{2}\right]=\rho A V_{N} D^{T} \int_{0}^{d} N^{T} N_{x^{\prime}} d x^{\prime} D} \\
& V_{N}=(1-(i-1) / N) i_{b} \\
& {\left[K_{v}\right]=-\left[K_{1}\right]-\left[K_{2}\right]-\left[K_{3}\right]-\left[K_{3}\right]^{T}} \\
& {\left[K_{1}\right]=\rho A i^{2} D^{T} \int_{0}^{T} N_{l}^{T} N_{l} d x^{\prime} D} \\
& {\left[K_{2}\right]=\rho A V_{N}^{2} D^{T} \int_{0}^{d} N_{x^{\prime}}^{T} N_{x^{\prime}} d x^{\prime} D} \\
& {\left[K_{3}\right]=\rho A i V_{N} D^{T} \int_{0}^{T} N_{l}^{T} N_{x^{\prime}} d x^{\prime} D}
\end{aligned}
$$

where $l$ is the length of the element, $i$ is the time derivatives, $i_{b}$ is the time derivatives of the whole beam length, $D$ is the coordinate transformation matrix from $\Delta q$ to $\Delta q^{\prime}$. The subscripts of $N$ indicate the partial derivatives by the variables. In the simulation the equations of motion for total system can be obtained by composing the mass, damping, and stiffness matrices of each element respectively in Eq.(8).

\subsection{Simulation results of the moving cantilever beam}

Table 1 Features using in the simulation

\begin{tabular}{|l|l|}
\hline Material & Steel \\
Length m m & 1.0 \\
Thickness m & $5.0 \times 10^{-3}$ \\
Cross Section Area m $\mathrm{m}^{2}$ & $5.0 \times 10^{-3}$ \\
Bending Rigidity $\mathrm{Nm}^{2}$ & $2.145 \times 10^{3}$ \\
\hline
\end{tabular}

Table 1 shows the features using in the simulation of the cantilever beam motion by using the present model as shown in Fig.2. The response of rolling material just after detaching from the right-side rolling stand as shown in Fig.1 is taken into consideration, then loads are assumed to be in step. Fig.4 shows the simulation results of the tip displacement of cantilever beam. From this figure it is shown that the beam is drawn into the constraint region with violent vibration as the beam becomes to be shorter. It is caused by the beam length to be short that the period of the vibration becomes to be short with respect to time.

\section{Dynamic characteristics using a simple model}

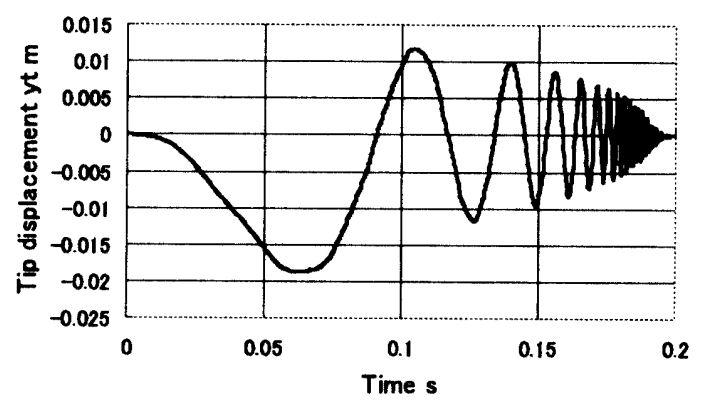

Fig.4 Tip displacement of cantilever beam moving along the axial direction

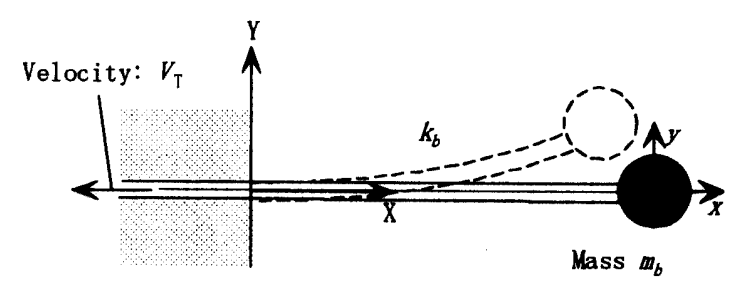

Fig.5 One mass model of cantilever beam

In this section the dynamic characteristics are examined using a simple model for the cantilever beam moving along the axial direction.

The cantilever beam is assumed to be one mass model as shown in Fig.5. The displacement $y$ in $Y$ direction and $x$ in axial direction at the tip point are considered as the degrees of freedom. The mass in Y direction $m_{b}$ and the stiffness $k_{b}$ of the cantilever beam can be obtained as follows:

$$
\begin{aligned}
& m_{b}=3 \rho A l_{b} / \alpha^{4}, \\
& k_{b}=3 E I / l_{b}{ }^{3} .
\end{aligned}
$$

where $\alpha$ is the non-dimensional number related to the natural frequency, $\alpha=1.875$ in the first mode of the cantilever beam. $l_{\mathrm{b}}$ is the beam length. Considering the beam length to be varied with respect to time, ignoring the higher order infinitesimal terms, Lagrange's function $L$ can be obtained as follows:

$$
L=\frac{1}{2} m_{b} \dot{y}^{2}+\frac{1}{2} \rho A l_{b} i_{b}^{2}-\frac{1}{2} k_{b} y^{2},
$$

where $\dot{y}, i_{\mathrm{b}}$ are time derivatives of $y, l_{\mathrm{b}}$ respectively. Substituting Eq.12 into Lagrange's equation of motion:

$$
\frac{\partial}{\partial t}\left(\frac{\partial L}{\partial \dot{y}}\right)-\frac{\partial L}{\partial y}=0
$$

the equation of motion is obtained as follows:

$$
m_{b} \ddot{y}+m_{b} \frac{i_{b}}{l_{b}} \dot{y}+k_{b} y=0 \text {. }
$$

As the coefficient of the second term in the left side of the above equation is to be negative when the beam is drawn at the constant speed: $i_{b}=-V_{T}$, the self-excited vibration can be induced. Substituting $y-Y^{\lambda t}$ into Eq.14, the discriminant of the characteristics equation in Eq.15 is derived as Eq.16. In the region of Eq.16, the natural frequency $\omega_{\mathrm{n}}$ and damping ratio $\zeta$ is obtained as Eq.17.

$$
\begin{aligned}
& m_{b} \lambda^{2}+m_{b} \frac{i_{b}}{l_{b}} \lambda+k_{b}=0 \\
& \left(m_{b} \frac{i_{b}}{l_{b}}\right)^{2}-4 m_{b} k_{b}<0 \\
& \omega_{n}=\frac{\alpha^{2}}{l_{b}^{2}} \sqrt{\frac{E I}{\rho A}}
\end{aligned}
$$




$$
\zeta=\frac{l_{b} i_{b}}{2 \alpha^{2}} \sqrt{\frac{\rho A}{E I}}
$$

The natural frequency is inversely proportional to the square of the length, and the damping ratio is proportional to the length in the case of the constant velocity. If the beam is drawn at the speed that the discriminant of the characteristics equation is positive, the eigenvalue can be positive real root, then the divergence can be induced.

\section{Relation between the damper-spring property at the support point and the system stability}

The vibration control technique for the cantilever beam moving along the axial direction is examined in this section. The vibration characteristics in varying damper-spring property at the support point of the cantilever beam are examined, and the optimum damper-spring property is obtained.

\subsection{Equations of motion}

In order to examine the effect of the damper-spring property at the support point of the cantilever beam, two masses model is considered as shown in Fig.6. The point $A$ is the tip point of the cantilever beam, and the displacement $y_{1}$ in Y-direction and the displacement $x$ in the axial direction are taken into consideration. The point $B$ is the support point assuming to be constrained in the rotational direction, and the displacement $y_{2}$ in Y-direction and the same displacement $x$ in the axial direction as the point $A$ are taken into consideration. The masses in Y-direction at points $A$ and $B$ are assumed to be onehalf of the total mass of the beam.

$$
m_{1}=m_{2}=\rho A l_{b} / 2
$$

Assuming the cantilever beam length to be varied with respect to time, and ignoring the higher order terms, Lagrange's function $L$ and the dissipation function $F$ are obtained as follows:

$L=\sum_{\mathrm{i}=1}^{2} \frac{1}{2} m_{\mathrm{i}} \dot{y}_{\mathrm{i}}^{2}+\frac{1}{2} \rho A l_{b} i_{b}^{2}-\frac{1}{2} k_{b}\left(y_{1}-y_{2}\right)^{2}-\frac{1}{2} k_{s} y_{2}^{2}$

$$
F=\frac{1}{2} c_{s} \dot{y}_{2}^{2}
$$

Substituting Eq.19,20 into Lagrange's equations of motion in Eq.21, the equations of motion in Eq.22 can be obtained as

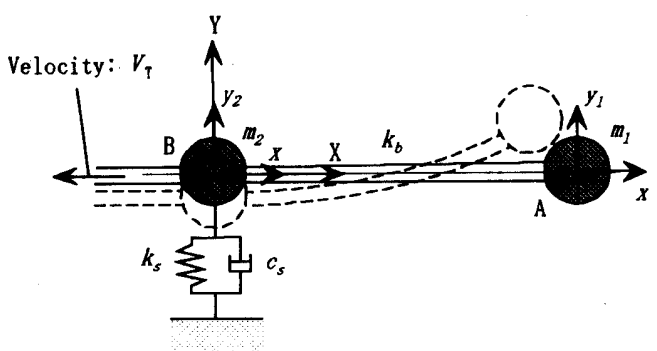

Fig.6 Two masses model of cantilever beam

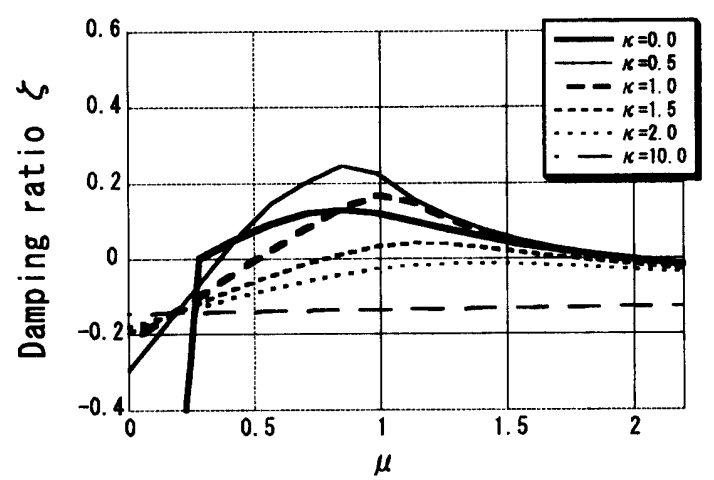

(a) $\quad l_{b}=1 m$

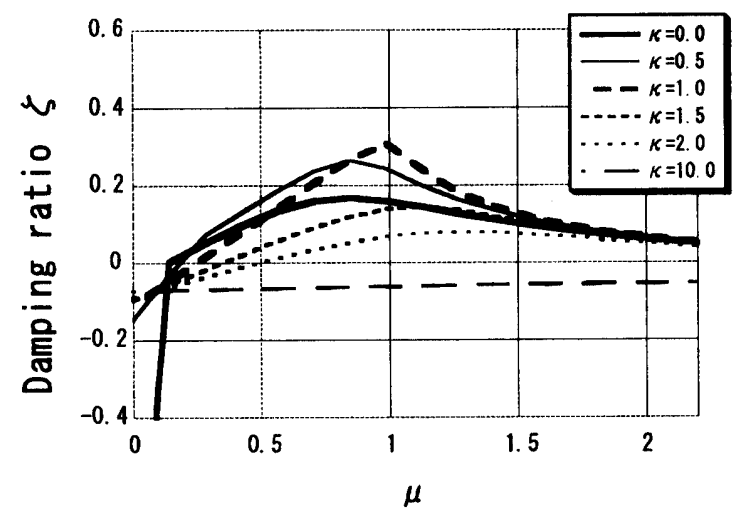

(b) $\quad l_{b}=0.5 m$

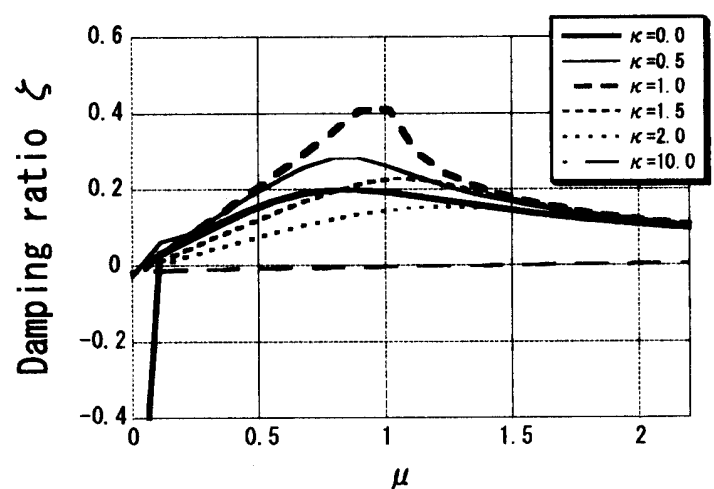

(c) $l_{b}=0.1 \mathrm{~m}$

Fig.7 Damping ratio $\zeta$ of the cantilever beam

follows:

$$
\frac{\partial}{\partial t}\left(\frac{\partial L}{\partial \dot{y}_{j}}\right)-\frac{\partial L}{\partial y_{j}}+\frac{\partial F}{\partial \dot{y}_{j}}=0(j=1,2)
$$




$$
\begin{gathered}
{\left[\begin{array}{cc}
m_{1} & 0 \\
0 & m_{2}
\end{array}\right]\left\{\begin{array}{l}
\ddot{y}_{1} \\
\ddot{y}_{2}
\end{array}\right\}+\left[\begin{array}{cc}
m_{1} \frac{i_{b}}{l_{b}} & 0 \\
0 & m_{2} \frac{i_{b}}{l_{b}}+c_{s}
\end{array}\right]\left\{\begin{array}{c}
\dot{y}_{1} \\
\dot{y}_{2}
\end{array}\right\}} \\
+\left[\begin{array}{cc}
k_{b} & -k_{b} \\
-k_{b} & k_{b}+k_{s}
\end{array}\right]\left\{\begin{array}{l}
y_{1} \\
y_{2}
\end{array}\right\}=0
\end{gathered}
$$

Substituting $\quad\left\{y_{1} y_{2}\right\}^{\mathrm{T}}-\left\{Y_{1} Y_{2}\right\}^{T} \mathrm{e}^{\lambda_{\mathrm{H}}}$ into Eq.22, the characteristics equations are obtained. The stability analysis of the system is conducted by complex eigenvalue analysis of Eq.23.

$$
\left|\begin{array}{cc}
\lambda^{2} m_{1}+\lambda m_{1} \frac{i_{b}}{l_{b}}+k_{b} & -k_{b} \\
-k_{b} & \lambda^{2} m_{2}+\lambda m_{2} \frac{i_{b}}{l_{b}}+\lambda c_{s}+k_{b}+k_{s}
\end{array}\right|=0
$$

\subsection{Evaluation of the stability}

In order to examine the effect of vibration control by the damper-spring at the support point of the cantilever beam, the system stability evaluation is carried out by the complex eigenvalue analysis. The features are used in the analysis as shown in Table 1. The non-dimensional parameters are employed to express the damper-spring property at the support point as follows:

$$
\mu=\frac{c_{s}}{2 \sqrt{m_{1} k_{b}}} \quad, \quad \kappa=\frac{k_{s}}{k_{b}}
$$

Firstly, the analyses are conducted using $\mu, \kappa$ in the length of the cantilever beam to be $l_{b}=1 \mathrm{~m}$. The damping ratios $\zeta$ obtaining from the complex eigenvalue analysis are shown in Fig.7(a). In the case of $\kappa=0$, the positive real roots generate in the region $\mu \leq 0.2$ where the divergence occurs, that is, $\zeta=-1$. Although the damping ratio $\zeta$ gradually becomes to be large in the region $0.2 \leq \mu \leq 0.8$, inversely to be small in the region $\mu \geq 0.8$, and the system becomes unstable in the region $\mu \geq 2$. As $\kappa$ turns to be large, $\zeta$ turns to be maximum in the region $0.5 \leq \kappa \leq 1.0$. The maximum $\zeta$ is obtained at $\mu=1$ in the case of $\kappa=1$. As $K$ turns to be greater than 1 , however, maximum

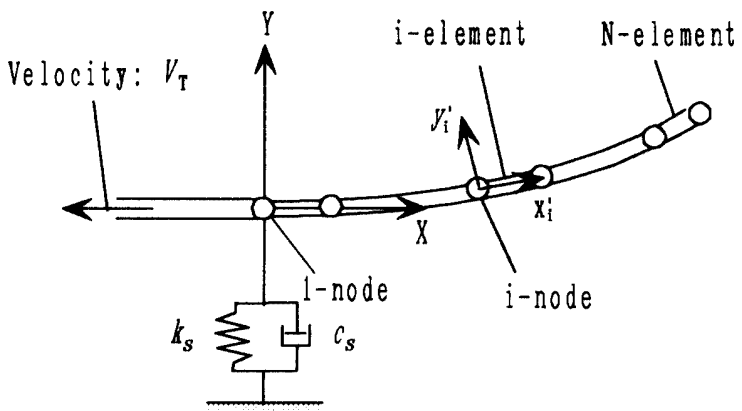

Fig.8 Cantilever beam model with a spring-damper support

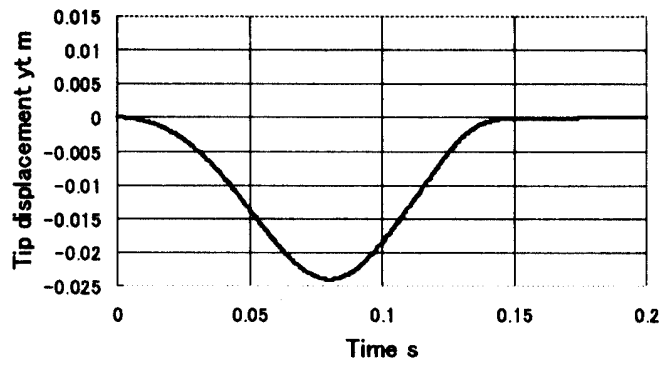

Fig.9 Tip displacement of cantilever beam with an optimum spring-damper support condition

$\zeta$ decrease, and $\zeta$ turns to be negative constantly in the region $x \geq 2$, then there is no stable condition.

Secondly, the similar analyses are conducted in the length of the cantilever beam to be $l_{b}=0.5,0.1 \mathrm{~m}$. The results are shown in Fig.7(b),(c). These results indicate in the same manner as Fig.7(a), and the stable region is expanded for $\mu, \kappa$. Especially, the maximum $\zeta$ is obtained nearly at $\mu=\kappa=1$.

\section{Evaluation of the vibration control method by using the finite element model}

In this section the vibration control method for the cantilever moving beam is examined considering the results obtained in the previous section. The effect is verified by using the detail finite element model as shown in Section 2.

5.1 In the case of damper-spring property at the support point to be variable

From the examination in the previous section, the vibration control method is proposed for the system to be stable that the damper- spring property at the support point is varied so as to be $\mu=\kappa=1$ in proportion to the cantilever beam length. That is, $c_{s}$ and $k_{s}$ are varied as the function of $l_{b}$ as follows:

$$
\begin{aligned}
& c_{s}=2 \sqrt{m_{1} k_{b}}=\frac{1}{l_{b}} \sqrt{6 \rho A E I} \\
& k_{s}=k_{b}=\frac{3 E I}{l_{b}{ }^{3}}
\end{aligned}
$$

The simulations for the cantilever beam that is drawn into the constraint region are carried out by using finite element model as shown in Section 2 in the case that the damperspring property is the function of the cantilever length. The effect of the vibration control is verified by the simulation. The analysis model is shown in Fig.8. The support point has one degree of freedom in Y-direction, and the damper-spring are set in the direction, while the rotational degree of freedom is fixed. Comparing Fig.9 with Fig.4, it is shown that the

Copyright (c) 2002 by JSME 
vibration control is conducted fairly and the beam is drawn without the vibration because the vibration energy is absorbed by the damper at the support point. The maximum displacement in Fig.9, however, becomes larger than that in Fig. 4 in order to support elastically at the support point.

5.2 Adaptive control of the damper property at the support point

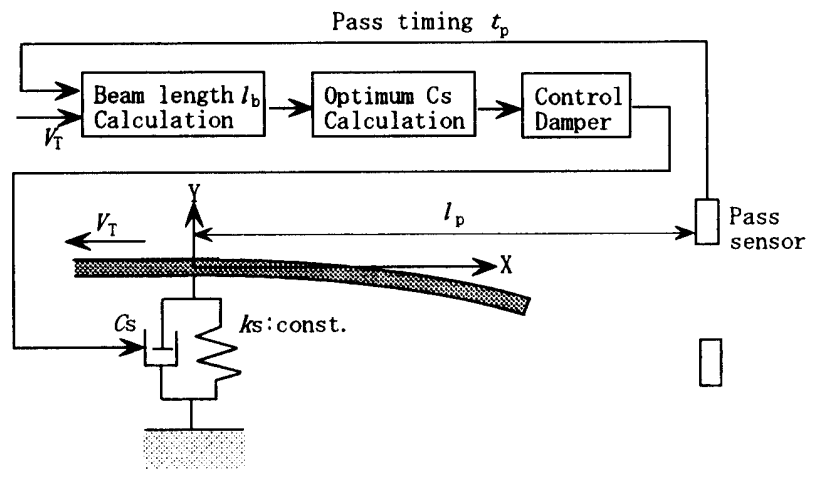

Fig.10 Vibration control system

In the vibration control actually, it is not so easy to control the spring property, while the control of the damping property is often used in general. Therefore, the control method is considered that the damper property at the support point is only controlled as shown in Fig.10.

In the control system, the timing $t_{\mathrm{p}}$ for the end of the beam to pass is detected by the sensor as shown in Fig.10, and the present length $l_{b}$ is obtained by Eq.26, then the damper is controlled so as to be the optimum damping ratio Cs given in Eq.25a.

$$
l_{b}=l_{p}-V_{T}\left(t-t_{p}\right)
$$

The simulations for the cantilever beam that is drawn into the constraint region are carried out by using the finite element model as shown in Section 2, and the results are shown in Fig.11. In order to obtain the optimal value for the stiffness of the spring at the support point, the simulation is conducted for

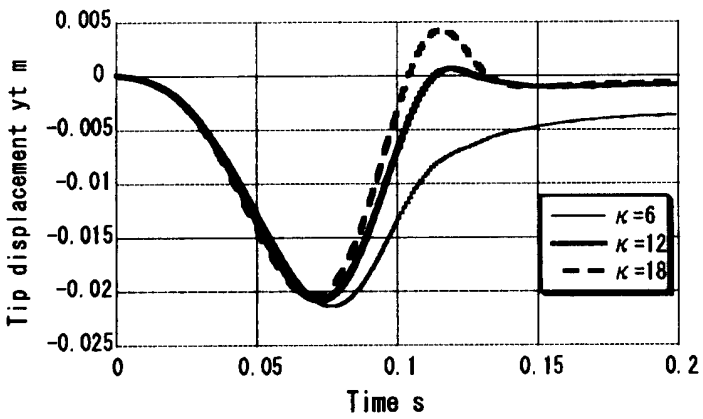

Fig.11 Tip displacement response (parameter: $\kappa$ ) the parameter $k_{\mathrm{s}}$. The $\kappa$ in Fig.11 indicates the ratio $k_{\mathrm{b}}$ to $k_{\mathrm{s}}$ in the initial condition. From this figure it is shown that the vibration control is conducted fairly in the condition $\kappa=12$.

Comparing these results to that in Fig.4,9, it is shown that excellent vibration control performance is obtained, while the overshoot is observed slightly in the condition $\kappa=12$.

\section{Conclusion}

In this paper the vibration of the cantilever beam moving along the axial direction that is observed for the plates and the wire rods in the mill is taken up, the vibration control method is proposed for the system, while examining the dynamic characteristics. From these results, the following conclusions are obtained.

(1) It is shown that the vibration of the cantilever beam moving along the axial direction is caused by self-excited vibration by examining the dynamic characteristics using the simple model.

(2) The optimal damper-spring property for controlling the cantilever beam vibration is obtained by examining the relation between damper-spring property at the support point and the system stability.

(3) The vibration control method is proposed for the system, and the effect of the vibration control is verified by numerical simulation using the finite element model. Finally, it is verified that the vibration of the beam are reduced by the proposed vibration control method.

\section{References}

[1] Stolte, J. and Benson, R.C., Dynamic Deflection of Paper Emerging from a Channel, Trans. ASME Journal of Vibration and Acoustics, 114(1992), pp.187-193.

[2] Yuh, J. and Young, T., Dynamic Modeling of an Axially Moving Beam in Rotation: Simulation and Experiment, Trans. ASME Journal of Dynamic Systems, Measurement, and Control, 113(1991), pp.34-40.

[3] Sugiyama, H. and Kobayashi, N., Analysis of Spaghetti Problem Using Multibody Dynamics, JSME, 65-631, C(1999), pp.910-915.

[4] Sugano, N., Imanishi, E. and Honke, K., Dynamic Analysis of Planar Beams Moving Along the Axial Direction, JSME, 67-653, C(2001-1), pp. 37-42. 